\title{
Simple Sequence Repeat Markers from Cercis canadensis Show Wide Cross-species Transfer and Use in Genetic Studies
}

\author{
Phillip A. Wadl ${ }^{1}$ and Robert N. Trigiano \\ Department of Entomology and Plant Pathology, University of Tennessee, 2431 Joe Johnson Drive, \\ 205 Ellington Plant Science Building, Knoxville, TN 37996
}

\author{
Dennis J. Werner \\ Department of Horticultural Science, North Carolina State University, Box 7609, Raleigh, NC 27695 \\ Margaret R. Pooler \\ U.S. Department of Agriculture-Agricultural Research Service, Floral and Nursery Plants Research \\ Unit, 10300 Baltimore Avenue, Building 010-A BARC-West, Beltsville, MD 20705
}

Timothy A. Rinehart

U.S. Department of Agriculture-Agricultural Research Service, Thad Cochran Southern Horticultural Laboratory, 810 Highway 26 West, Poplarville, MS 39470

\begin{abstract}
AdDitional INDEX words. microsatellites, redbud, SSR, woody ornamental tree
Abstract. There are 11 recognized Cercis L. species, but identification is problematic using morphological characters, which are largely quantitative and continuous. Previous studies have combined morphological and molecular data to resolve taxonomic questions about geographic distribution of Cercis species, identifying botanical varieties, and associations between morphological variation and the environment. Three species have been used in ornamental plant breeding in the United States, including three botanical varieties of $C$. canadensis $L$. from North America and two Asian species, $C$. chingii Chun and $C$. chinensis Bunge. In this article, 51 taxa were sampled comprising eight species of Cercis and a closely related species, Bauhinia faberi Oliv. Sixty-eight polymorphic simple sequence repeat markers were used to assess genetic relationships between species and cultivars. For all samples the number of alleles detected ranged from two to 20 and 10 or more alleles were detected at 22 loci. Average polymorphic information content was 0.57 and values ranged from 0.06 to 0.91 with 44 loci 0.50 or greater. Cross-species transfer within Cercis was extremely high with 55 loci that amplified at $100 \%$. Results support previously reported phylogenetic relationships of the North American and western Eurasian species and indicate suitability of these markers for mapping studies involving $C$. canadensis and $C$. chinensis. Results also support known pedigrees from ornamental tree breeding programs for the widely cultivated $C$. canadensis and $C$. chinensis species, which comprised the majority of the samples analyzed.
\end{abstract}

Cercis canadensis, a leguminous tree, is native to North America and cultivated widely as an ornamental. Flowers emerge directly from the stem or trunk before the leaves early in the spring. Petal colors range from purple to pink to red or white. A double-flowered cultivar is available. Growth habits include weeping forms, dwarf types, and small- to medium-sized types. Leaves can be glossy to pubescent and leaf color from green to purple or variegated. There are more than three dozen $C$. canadensis cultivars commercially available in the United States that encompass the major phenotypic variants. Ornamental traits are usually simply inherited, and novel combinations of traits are expected from breeding and selection (Werner, 2006).

Received for publication 2 Apr. 2012. Accepted for publication 3 May 2012. This work was supported by U.S. Department of Agriculture Grant \#58-6404-7-213. Mention of trade names or commercial products in this article is solely for the purpose of providing specific information and does not imply recommendation or endorsement by the University of Tennessee, North Carolina State University, or U.S. Department of Agriculture.

We thank Mark Weathington of the JC Raulston Arboretum at North Carolina State University and Holly Forbes of the University of California-Berkeley Botanic Garden for kindly providing material for this study.

${ }^{1}$ Corresponding author. E-mail: pwadl@utk.edu.
There are three recognized botanical varieties of $C$. canadensis, which account for the high degree of morphological variation in the cultivated forms (Isley, 1975). C. canadensis var. canadensis is found in the eastern United States and is noted for dull green leaves with acute apices. It has glabrous branchlets and leaves that can be glabrous or pubescent. C. canadensis var. texensis (S. Watson) M. Hopkins grows in Texas and Oklahoma and has thick, glossy leaves and branchlets that are also glabrous. C. canadensis var. mexicana (Rose) M. Hopkins is found in northern Mexico and southern Texas. Leaves are thick and shiny with rounded apices with branchlets that are pubescent. In an effort to resolve the phylogeny, several taxonomic studies were previously conducted with morphological characters and DNA sequences (Davis et al., 2002). Most of the morphological characters are continuous over the geographic ranges, and variation, particularly in leaf morphology, may be a response to different climatic regions (Davis et al., 2002). Characters such as pubescence are thought to have evolved along temperature and moisture clines (Fritsch et al., 2009). DNA studies support geographic distributions, but internal transcribed spacer (ITS) sequence data have low resolution within the populations sampled (Davis et al., 2002; Fritsch and Cruz, 2012). 
In situations in which botanical varieties of $C$. canadensis are growing side by side in the wild, little introgression is recorded, possibly as a result of differences in timing of flowering. However, all three botanical varieties are sexually compatible as evidenced by complex controlled hybridizations in ornamental breeding programs (Werner, 2006). In cultivated forms, variation in leaf traits generally corresponds to genetic background; however, introgression of traits among these three botanical varieties is becoming more common. Complex pedigrees make taxonomic placement relatively ambiguous based on morphological characters alone, although true var. canadensis forms are typically determined by their dull, thin leaves. Although not widely grown as an ornamental tree, C. occidentalis Torr. Ex A. Gray also is native to the North America, including parts of California, Arizona, Utah, and Nevada (Isley, 1975).

Two other species of Cercis, both native to Asia, are cultivated as ornamental plants in the United States. C. chingii is found in southeastern China in mixed temperate or warm forests and C. chinensi is widely distributed in southern China (Li, 1944). Both species have desirable ornamental traits. $C$. chinensis, commonly known as chinese redbud, grows quickly and produces flowers at an earlier age than other species in production. It has prolific bud set and a growth habit of a shrub or compact small tree. $C$. chingii has large pink flowers that are the first to open of all of the redbuds (Dirr, 1998). There are five additional Cercis species found in Asia including C. griffithii Bioss, C. glabra Pamp., C. gigantea F.C. \& Keng f., C. chuniana F.P. Metcalf, and C. racemosa Oliv. (Hopkins, 1942). C. siliquastrum L. is found in the Mediterranean region from France to Turkey (Hopkins, 1942; Isley, 1975). All species except $C$. chuniana are represented in the study presented here.

Genetic relatedness, particularly among botanical varieties and species, is an important consideration when estimating the use and practicality of wide hybridizations in ornamental plant breeding. Continuous, qualitative, and wide-ranging morphological traits are an important source of variation for plant breeding programs but are not the most useful characters for phylogenetic estimates. DNA sequences such as the nuclear ribosomal ITS region have proven useful in resolving phylogenetic questions about plant species (for review, see Baldwin et al., 1995). However, ITS sequences are less useful in plant breeding when confirming pedigrees, establishing markers for linkage to important traits, and genetic mapping. In this respect, molecular markers that are randomly dispersed throughout the nuclear genome have the required distribution and frequency to provide information at the population and single plant levels. Simple sequence repeat (SSR) markers are widely used because of their high repeatability between laboratories, codominant nature, and potential transferability across related species.

The objectives of this study were to use SSRs from $C$. canadensis to accomplish the following: 1) determine the cross-species transfer within Cercis; 2) independently reconstruct species relationships within Cercis using SSR data and ITS sequence data; and 3) address at what taxonomic level the SSR loci are useful for breeding and genetics within Cercis in the context of the results.

\section{Materials and Methods}

Plant material and DNA extraction. In this study, the following eight species of Cercis and a closely related genus were analyzed: C. canadensis (26), C. canadensis var. mexicana (one), C. canadensis var. texensis (three), C. chinensis (eight), C. chingii (three), C. gigantea (one), C. glabra (two), C. griffithii (one), C. occidentalis (one), C. racemosa (one), C. siliquastrum (three), and B. faberi (one), for a total of 51 individuals (Table 1). Genomic DNA was extracted from $1 \mathrm{~cm} \times 1-\mathrm{cm}$ pieces of fresh leaf tissue using the Qiagen Plant Mini Kit (Qiagen, Valencia, CA) and quantified using a spectrophotometer (NanoDrop; Thermo Fisher Scientific, Waltham, MA).

SiMPLE SEQUENCE REPEAT MARKERS. To assess transferability of SSRs from C. canadensis, six genomic SSRs (Rinehart et al., 2010) and an additional 62 SSRs reported here were used to amplify DNA of 51 Cercis samples (Table 2). Development of the 62 SSRs was as described by Rinehart et al. (2010). All SSRs used were single locus and 58 of the loci were polymorphic in C. canadensis.

For polymerase chain reaction (PCR), forward primers were $5^{\prime}$ tailed with the sequence 5'-CAGTTTTCCCAGTCACGAC-3' (Waldbieser et al., 2003) to permit product labeling, and reverse primers were tailed at the $5^{\prime}$ end with the sequence $5^{\prime}$-GTTT- $3^{\prime}$ to promote non-template adenylation (Brownstein et al., 1996). A primer, 5'-CAGTTTTCCCAGTCACGAC-3', was labeled with 5-carboxy-fluorescein (Integrated DNA Technologies, Coralville, IA) and added to the amplification reaction, which included Advantage2 Taq DNA Polymerase (Clontech, Mountain View, CA) according to previously published protocols (Rinehart et al., 2006). Fluorescence-labeled PCR fragments were visualized by automated capillary gel electrophoresis using dye set 32 on an ABI 3130xl using ROX-500 size standard (Applied Biosystems, Carlsbad, CA). GeneMapper Version 4.0 was used to identify and assign allele sizes (Applied Biosystems).

AMPLIFICATION AND SEQUENCING OF THE INTERNAL TRANSCRIBED SPACER REGION. The ITS regions, including the 5.8S nuclear rDNA gene, for accessions from 13 Cercis and one B. faberi (outgroup) samples (indicated with an asterisk in Table 1) were amplified using the primers ITS1 and ITS4 (White et al., 1990). Reaction mixtures contained $40 \mathrm{ng}$ DNA, $0.5 \mathrm{~mm}$ of each primer, $1.5 \mathrm{~mm}$

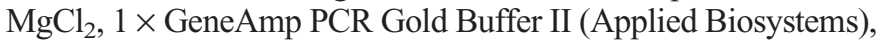
$0.2 \mathrm{~mm}$ dNTPs, $5 \%$ dimethyl sulfoxide, $1.2 \mathrm{U}$ of AmpliTaq Gold DNA polymerase (Applied Biosystems), and sterile water to $30 \mu \mathrm{L}$. Amplification was completed in a thermal cycler (Eppendorf, Hamburg, Germany) programmed for $2 \mathrm{~min}$ at 94 ${ }^{\circ} \mathrm{C}$ followed by 35 cycles of $30 \mathrm{~s}$ at $94^{\circ} \mathrm{C}, 1 \mathrm{~min}$ at $57^{\circ} \mathrm{C}$, and 1 min at $72{ }^{\circ} \mathrm{C}$ with a final $7 \mathrm{~min}$ at $72^{\circ} \mathrm{C}$. A single PCR product was observed for all amplifications when visualized on a $2 \%$ agarose gel stained with ethidium bromide. The ITS PCR products were first cleaned with the QIAquick PCR Purification Kit (Qiagen) and then cloned with the pGEM-T Easy Vector System II (Promega, Madison, WI). Plasmid DNA was isolated using the QIAprep Miniprep Kit (Qiagen) and sequenced using ABI Big-Dye Version 3.1 terminators (Applied Biosystems) on an ABI 3730 Genetic Analyzer. Two random clones per accession were sequenced to control for sequence polymorphisms and in total 28 copies of the ITS region were sequenced. Sequences from opposing strands were reconciled and verified for accuracy using Sequencher 4.2.2 (Gene Codes, Ann Arbor, MI) and are archived in GenBank.

DAta ANAlysis of Simple SEQUence Repeats. Data from 68 SSR loci were compiled for the 51 individuals. Summary statistics were generated using Cervus software including calculations for the number of alleles, polymorphic information content (PIC), and the frequency of null alleles (Kalinowski et al., 2007). To determine species relationships within Cercis, the program Populations Version 1.2.30 (Langella, 2002) was 
Table 1. Cercis accessions and cultivars that were analyzed using 68 simple sequence repeat (SSR) loci. ${ }^{\mathrm{z}}$

\begin{tabular}{|c|c|c|}
\hline Species or hybrid & Native range & Cultivar or accession no. \\
\hline Bauhinia faberi & Asia (China, Laos, Myanmar, Thailand) & $88.1198^{\mathrm{y}, \mathrm{u}}$ \\
\hline Cercis canadensis & $\begin{array}{l}\text { Eastern North America (Connecticut } \\
\text { to Nebraska, south to Florida) }\end{array}$ & $\begin{array}{l}\text { 'Ace of Hearts', 'Alba', 'Appalachia Red', } \\
\text { 'Cascading Hearts', 'Covey', 'Crosswick's Red', } \\
\text { 'Flame', 'Floating Clouds', 'Forest Pansy', 'Greswan', } \\
\text { 'Hearts of Gold', 'High Country Gold', 'JN2', 'JN3', } \\
\text { 'Little Woody', 'Mardi Gras', 'Merlot', 'Morton', } \\
\text { 'Pauline Lily', 'Pink Heartbreaker', 'Royal White', } \\
\text { 'Ruby Falls', 'Rubye Atkinson', 'Silver Cloud', } \\
\text { 'Tennessee Pink', 'Woody' }\end{array}$ \\
\hline $\begin{array}{l}\text { C. canadensis } \\
\text { var. mexicana }\end{array}$ & $\begin{array}{l}\text { Southwestern North America (Edwards Plateau and } \\
\text { southward to the northern Sierra Madre Oriental) }\end{array}$ & NA65244 \\
\hline $\begin{array}{l}\text { C. canadensis } \\
\text { var. texensis }\end{array}$ & $\begin{array}{l}\text { Southwestern North America (Edwards Plateau and } \\
\text { southward to the northern Sierra Madre Oriental) }\end{array}$ & 'Oklahoma', 'Texas White', 'Traveller' \\
\hline C. chinensis & $\begin{array}{l}\text { China (Anhui, Fujian, Guangdong, Guangxi, Guizhou, } \\
\text { Hebei, Henan, Hubei, Hunan, Jiangsu, Liaoning, } \\
\text { Shaanxi, Shandong, Shanxi, Sichuan, Yunnan, } \\
\text { Zhejiang) }\end{array}$ & $\begin{array}{l}\text { 92.1455, y,u ‘Avondale', 'Azalea Alba', 'Christata', } \\
\text { 'Don Egolf', 'Genpei', 'Shibamichi Red', 'Shirobana' }\end{array}$ \\
\hline C. gigantea $v$ & $\begin{array}{l}\text { China (Anhui, Guangdong, Guangxi, Guizhou, Henan, } \\
\text { Hubei, Hunan, Shaanxi, Sichuan, Yunnan, Zhejiang) }\end{array}$ & NA49082 (94-1EC) $)^{\mathrm{x}, \mathrm{u}}$ \\
\hline C. glabra & $\begin{array}{l}\text { China (Anhui, Guangdong, Guangxi, Guizhou, Henan, } \\
\text { Hubei, Hunan, Shaanxi, Sichuan, Yunnan, Zhejiang) }\end{array}$ & 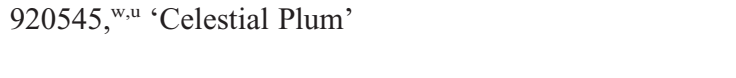 \\
\hline C. griffithii & India, Pakistan, Tajikistan, Uzbekistan & $980329^{\mathrm{w}, \mathrm{u}}$ \\
\hline C. occidentalis & $\begin{array}{l}\text { Western North America (Arizona, California, } \\
\text { Nevada, Utah) }\end{array}$ & $55.0508^{\mathrm{y}, \mathrm{u}}$ \\
\hline C. racemosa & $\begin{array}{l}\text { China (W Guizhou, W Hubei, Hunan, Shaanxi, } \\
\text { E Sichuan, Yunnan) }\end{array}$ & $80062^{\mathrm{w}, \mathrm{u}}$ \\
\hline C. siliquastrum & $\begin{array}{l}\text { Mediterranean region (France to Turkey } \\
\text { and Afghanistan) }\end{array}$ & 89.0953 , y 20080, w,u 'Bodnant' \\
\hline
\end{tabular}

${ }^{\mathrm{z}}$ Samples were also used for internal transcribed spacer (ITS) region sequence analysis.

yUniversity of California-Berkeley Botanic Garden, Berkeley, CA.

${ }^{x}$ U.S. National Arboretum, Washington, DC.

wJC Raulston Arboretum at North Carolina State University, Raleigh, NC.

vPlants may represent a distinct species, but too little material was available to be certain (Dezhao et al., 2010).

"GenBank accession numbers for ITS sequence analysis: B. faberi $88.1198=\mathrm{JQ425121}$, C. canadensis 'Ace of Hearts' = JQ425128, C. canadensis var. mexicana NA65244 = JQ425119, C. canadensis var. texensis 'Oklahoma' = JQ425129, C. chinensis $92.1455=\mathrm{JQ} 425120$, $C$. chingii NA54099 = JQ425125, C. chingii $20086=\mathrm{JQ425130,} \mathrm{C.} \mathrm{chingii} 100143=\mathrm{JQ425123,} \mathrm{C.} \mathrm{gigantea} \mathrm{NA49082} \mathrm{=} \mathrm{JQ425131,} \mathrm{C.} \mathrm{glabra}$

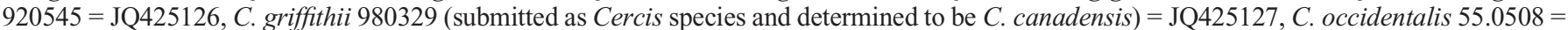
JQ425124, C. racemosa $80062=\mathrm{JQ425132,} \mathrm{C.} \mathrm{siliquastrum} 20080=\mathrm{JQ} 425122$.

used to calculate two separate genetic distance matrices based on the methods of Nei et al. (1983). One matrix contained all 51 individuals and the other matrix consisted of 14 individuals (indicated with the letter $\mathrm{z}$ in Table 1). Unweighted pair group method with arithmetic mean (UPGMA) with 500 bootstrap replicates was used to generate the two dendrograms of genetic relationships. B. faberi was included as an outgroup for rooting the dendrograms, which were visualized and edited using TreeView Version 1.6.6 (Page, 1996).

\section{Results}

Genetic diversity of CerCIS AND CROSS-SPECIES/GENERA TRANSFER. Fifty-one taxa, comprising eight species of Cercis and the closely related B. faberi, were amplified with 68 polymorphic SSRs (Table 1). All loci used in this study were perfect di- to hexanucleotide repeats and were multiallelic. Genetic diversity was assessed by calculating the number of alleles and PIC (Table 2). The number of alleles detected for all samples ranged from two (loci 272a, 581c, 732a, 877a) to 20 (loci 461a, 980a), and 22 loci (32\%) detected 10 or more alleles. The average PIC was 0.57 and values ranged from 0.06 (locus 581 c) to 0.91 (locus 461a) with 44 loci 0.50 or greater.

Cross-species transfer of SSRs from $C$. canadensis var. canadensis to related Cercis species and B. faberi was determined by calculating for each locus the number of alleles, number of genotypes, and the percent transfer to other Cercis species (Table 3). Ten loci amplified in B. faberi and only one locus (324a) was heterozygous. For $C$. canadensis var. canadensis, the number of alleles ranged from one (locus 157a) to 14 (loci 461a, 671a, and 921a) and the number of genotypes detected ranged from one (locus 157a) to 23 (locus 921a). All except six loci (3a, 165a, 168a, 176a, 579a, and 871a) amplified in C. canadensis var. mexicana, and 16 of the loci that amplified were heterozygous. Only one locus (3a) did not amplify in C. canadensis var. texensis; the number of alleles detected ranged from one to five and 24 loci successfully distinguished 'Oklahoma', 'Texas White', and 'Traveller'. For the $C$. chinensis samples, one locus 
Table 2. Characteristics of 68 simple sequence repeat (SSR) loci developed from Cercis canadensis. ${ }^{\mathrm{z}}$

\begin{tabular}{|c|c|c|c|c|c|c|c|c|}
\hline Locus & $\begin{array}{c}\text { GenBank } \\
\text { accession no. }\end{array}$ & Primer sequence $\left(5^{\prime}-3^{\prime}\right)$ & Repeat & $\begin{array}{c}\text { Size } \\
\text { range (bp) }\end{array}$ & $\begin{array}{l}\text { Alleles } \\
\text { (no.) }\end{array}$ & PIC & $\begin{array}{l}\text { Alleles (no.) } \\
\text { C. canadensis }\end{array}$ & PIC \\
\hline $1006 \mathrm{a}$ & GU253212 & $\begin{array}{l}\text { F:GAGTCCACTGATTGCGGCAC } \\
\text { R:TTTGACAACATAGGAACAGAGAGTTGG }\end{array}$ & $(\mathrm{ACT})_{6}$ & $107-123$ & 6 & 0.57 & 3 & 0.23 \\
\hline $1057 \mathrm{a}$ & GU171393 & $\begin{array}{l}\text { F:TCCCTCTCAGCTTTCATATAATCCAC } \\
\text { R:AAAGAGAGATCGTTTAGAAGGCGG }\end{array}$ & $(\mathrm{CCATCA})_{7}$ & $120-175$ & 11 & 0.77 & 9 & 0.80 \\
\hline $1240 \mathrm{~b}$ & GU253251 & $\begin{array}{l}\text { F:TCTACGCCATTCCTCTTCATCTTC } \\
\text { R:GTGCTTTCAGTCATGGTGATTTTG }\end{array}$ & $(\mathrm{CT})_{4}$ & $103-127$ & 6 & 0.52 & 3 & 0.21 \\
\hline $127 \mathrm{spa}$ & GU252892 & $\begin{array}{l}\text { F:CCAATTCAATTCCTCTGTGTGTTG } \\
\text { R:AACGGTGTGACTAGGAGTCAAAGG }\end{array}$ & $(\mathrm{TC})_{4}$ & $87-110$ & 10 & 0.68 & 6 & 0.44 \\
\hline $136 \mathrm{a}$ & GU252897 & $\begin{array}{l}\text { F:TGGTTGACAAACTTTACATGGTGTG } \\
\text { R:TGCTTAATCGTTCTAATTGTTCCCC }\end{array}$ & $(\mathrm{CT})_{8}$ & $99-115$ & 8 & 0.54 & 4 & 0.15 \\
\hline $157 \mathrm{a}$ & GU252908 & $\begin{array}{l}\text { F:ACTTGCAACCCTTCTCCTGTATCC } \\
\text { R:ACGTGGGTGGGTGTAAGTATATGG }\end{array}$ & $(\mathrm{CT})_{4}$ & $139-158$ & 3 & 0.14 & 1 & 0.00 \\
\hline $164 a$ & GU252911 & $\begin{array}{l}\text { F:CCTCTAAAACTCAGGGTGCACTTTAC } \\
\text { R:CAATATGAATACCATCGAAGCCTG }\end{array}$ & $(\mathrm{AAAT})_{6}$ & $119-136$ & 8 & 0.62 & 6 & 0.44 \\
\hline $165 \mathrm{a}$ & GU252912 & $\begin{array}{l}\text { F:TGTTGCAGGTAAGTTATGCTTTGC } \\
\text { R:GAAGAAGCATGGAAACAGAAATGG }\end{array}$ & $(\mathrm{CTT})_{5}$ & $79-96$ & 7 & 0.61 & 5 & 0.35 \\
\hline $168 \mathrm{a}$ & GU252915 & $\begin{array}{l}\text { F:AACAAAAGCAAAAGCACGCTACTC } \\
\text { R:CAGTTGCCAAAATCAGAGAAATTG }\end{array}$ & $(\mathrm{CT})_{7}$ & $151-164$ & 4 & 0.41 & 3 & 0.46 \\
\hline $173 a$ & GU252917 & $\begin{array}{l}\text { F:GTGTTAGTTCTAGCGGGACAGGTG } \\
\text { R:CTTGTGCTTGTTGATTCCCAGAC }\end{array}$ & $(\text { TAGTGG })_{4}$ & $138-157$ & 4 & 0.41 & 3 & 0.40 \\
\hline $176 a$ & GU252918 & $\begin{array}{l}\text { F:GACTGCAAATCGGAAAGAAAGAAG } \\
\text { R:TCCTTCAGGGATGCATTTAAGAAC }\end{array}$ & $(A G)_{6}$ & $137-146$ & 5 & 0.47 & 3 & 0.40 \\
\hline $177 b$ & GU252919 & $\begin{array}{l}\text { F:AGAAATTTCAGAGACCGTGAGGTG } \\
\text { R:TAACACACTATCCGTCATTCCCAG }\end{array}$ & $(\mathrm{GA})_{6}$ & $154-183$ & 8 & 0.56 & 2 & 0.37 \\
\hline $178 \mathrm{a}$ & GU252920 & $\begin{array}{l}\text { F:AATGAAGAGGTTTGTGTCTGGTGC } \\
\text { R:ACGTATCTCTTGTCCAACCCAGAG }\end{array}$ & $(\mathrm{TC})_{4}$ & $113-119$ & 6 & 0.61 & 4 & 0.32 \\
\hline $180 \mathrm{a}$ & GU252921 & $\begin{array}{l}\text { F:GATTTCAAAGCCCTGTTAGTGCC } \\
\text { R:TTATTGTGTTTGTGAAGTAGCGCC }\end{array}$ & $(\mathrm{CT})_{5}$ & $83-104$ & 7 & 0.69 & 7 & 0.62 \\
\hline $199 a$ & GU252924 & $\begin{array}{l}\text { F:AATAACTCCTGGAACAATGGAGGG } \\
\text { R:TCTATGGTTTAGACCCTTTGTCACATC }\end{array}$ & $(\mathrm{GAGA})_{8}$ & $149-173$ & 8 & 0.40 & 3 & 0.28 \\
\hline $220 \mathrm{a}$ & GU252932 & $\begin{array}{l}\text { F:ACCCATTCACTACCGTTCATTGAG } \\
\text { R:GATTCCAGATTGTCACACGTTTTG }\end{array}$ & $(\mathrm{TATT})_{4}$ & $100-117$ & 5 & 0.56 & 4 & 0.28 \\
\hline $221 \mathrm{a}$ & GU252933 & $\begin{array}{l}\text { F:ACATTTTCCCAGAAGGTTGTTCTC } \\
\text { R:CCAACATCCATGATTTTGTGTG }\end{array}$ & $(\mathrm{TCTC})_{10}$ & $115-146$ & 9 & 0.52 & 6 & 0.42 \\
\hline $229 a$ & GU252937 & $\begin{array}{l}\text { F:CTGAGGTCCGAATGGTAATTGAAC } \\
\text { R:CGATAATACTCGATATATGCATTGCG }\end{array}$ & $(\mathrm{GAGAG})_{4}$ & $147-166$ & 8 & 0.56 & 2 & 0.17 \\
\hline $254 a^{z}$ & GU171389 & $\begin{array}{l}\text { F:CTCTCCCTCAACACTTGCAGTACC } \\
\text { R:TCCTGCTTTTAACATCGACGATTC }\end{array}$ & $(\mathrm{AG})_{12}$ & $130-154$ & 12 & 0.79 & 5 & 0.48 \\
\hline $268 \mathrm{a}$ & GU252951 & $\begin{array}{l}\text { F:GCTGGTGTGACAAACCTCAAAAC } \\
\text { R:GGTTCTAAGCAAGGTGAGCAAGAG }\end{array}$ & $(\mathrm{TC})_{4}$ & $133-160$ & 8 & 0.68 & 4 & 0.39 \\
\hline $272 a$ & GU252953 & $\begin{array}{l}\text { F:AAGCATTGAAGAAGAAAATGGGTG } \\
\text { R:TCAATTCCTCAGAACCCATAAACC }\end{array}$ & $(\mathrm{GA})_{4}$ & $70-80$ & 2 & 0.29 & 2 & 0.06 \\
\hline $287 b$ & GU252957 & $\begin{array}{l}\text { F:GTGATTGGGATGAGCCATAATTTC } \\
\text { R:ACCAATCCCAGTTACTTGAATAGAGG }\end{array}$ & $(\mathrm{GA})_{6}$ & $136-156$ & 7 & 0.52 & 3 & 0.12 \\
\hline $324 a$ & GU171390 & $\begin{array}{l}\text { F:CCGAGATCTGAATTACATAGAATATGCC } \\
\text { R:GCCAAAGCAAAACGAAAATATCAG }\end{array}$ & $(\mathrm{AG})_{8}$ & $137-159$ & 12 & 0.81 & 8 & 0.65 \\
\hline $37 a^{y}$ & GU171387 & $\begin{array}{l}\text { F:AAAGGGTAAGTTGCTGGAGGAGAC } \\
\text { R:ATTTGTGTTGACATGGCACCTTAG }\end{array}$ & $(\mathrm{GA})_{5}$ & $91-125$ & 16 & 0.82 & 8 & 0.62 \\
\hline $386 b$ & GU252998 & $\begin{array}{l}\text { F:ATTCGAATCTCTCTTGATTGCTCC } \\
\text { R:ACAGGAGACAAAAGACAGGGAAAG }\end{array}$ & $(\mathrm{TC})_{4}$ & $135-157$ & 5 & 0.60 & 3 & 0.32 \\
\hline $3 a$ & GU252837 & $\begin{array}{l}\text { F:AAAATCTGCTGACGTGGCATC } \\
\text { R:GTTTAACATGCAAAGCCAAAGAGG }\end{array}$ & $(\mathrm{TC})_{10}$ & $104-133$ & 14 & 0.88 & 10 & 0.83 \\
\hline $402 \mathrm{a}$ & GU253006 & $\begin{array}{l}\text { F:TGGATGTAATTAAAGAAGCGAGCC } \\
\text { R:GGGAGTTACAAAACAAAGCCATTC }\end{array}$ & $(\mathrm{CT})_{8}$ & $119-152$ & 11 & 0.44 & 4 & 0.28 \\
\hline $416 a$ & GU253010 & $\begin{array}{l}\text { F:CTTGGACTTTATCGACTCCACCAG } \\
\text { R:GAAAACGAAAAGCCAACTGAAATG }\end{array}$ & $(\mathrm{TCTTCT})_{7}$ & $117-134$ & 7 & 0.56 & 4 & 0.18 \\
\hline
\end{tabular}


Table 2. Continued.

\begin{tabular}{|c|c|c|c|c|c|c|c|c|}
\hline Locus & $\begin{array}{c}\text { GenBank } \\
\text { accession no. }\end{array}$ & Primer sequence $\left(5^{\prime}-3^{\prime}\right)$ & Repeat & $\begin{array}{c}\text { Size } \\
\text { range (bp) }\end{array}$ & $\begin{array}{l}\text { Alleles } \\
\text { (no.) }\end{array}$ & PIC & $\begin{array}{l}\text { Alleles (no.) } \\
\text { C. canadensis }\end{array}$ & PIC \\
\hline $441 \mathrm{a}$ & GU253023 & $\begin{array}{l}\text { F:GTCTGCATCGTTCAATTTCTGGAC } \\
\text { R:AGAATGGCGGGACTCTAAACTTTG }\end{array}$ & $(\mathrm{AG})_{12}$ & $74-93$ & 9 & 0.78 & 6 & 0.67 \\
\hline $461 \mathrm{a}$ & GU253032 & $\begin{array}{l}\text { F:TCACAAACAGGAAAAGGGAAGATG } \\
\text { R:CATTTCAGAATTCAGAGCAATCCC }\end{array}$ & $(\mathrm{AG})_{12}$ & $130-179$ & 21 & 0.91 & 16 & 0.90 \\
\hline $475 \mathrm{a}$ & GU253036 & $\begin{array}{l}\text { F:AGATAGCGACCGAGAAAATCAGAG } \\
\text { R:ACACCTCTTTCTCCCTTGAAGTCC }\end{array}$ & $(\mathrm{GAA})_{5}$ & $120-145$ & 6 & 0.50 & 3 & 0.23 \\
\hline $508 \mathrm{a}$ & GU253044 & $\begin{array}{l}\text { F:TATGCAGTGAGAAGGAAATGTTGG } \\
\text { R:AGTTACAGCAACAAGCAGGAGGAG }\end{array}$ & $(\mathrm{GAA})_{5}$ & $140-149$ & 4 & 0.32 & 3 & 0.40 \\
\hline $519 a$ & GU253048 & $\begin{array}{l}\text { F:TCAGTGTTATGATGCTCCATGTGAC } \\
\text { R:ATTATCTCATTGCCACCAACAACC }\end{array}$ & $(\text { TATC })_{5}$ & $149-169$ & 4 & 0.44 & 3 & 0.38 \\
\hline $53 \mathrm{a}$ & GU252855 & $\begin{array}{l}\text { F:TCCTTTGCTCATGGTAGTCTGATG } \\
\text { R:GCACTAAAGAGTTGTGTTCATGCC }\end{array}$ & $(\mathrm{AAAT})_{6}$ & $128-161$ & 15 & 0.87 & 8 & 0.74 \\
\hline $563 a$ & GU253065 & $\begin{array}{l}\text { F:TGTCAACACTCAACGATGGTAGC } \\
\text { R:TTTCAACCGTGTAAGTCATAAAGATTTC }\end{array}$ & $(\mathrm{TAC})_{7}$ & $115-167$ & 11 & 0.77 & 5 & 0.53 \\
\hline $579 a$ & GU253071 & $\begin{array}{l}\text { F:GGAAAAGGATTGAGAAACAAAAGTG } \\
\text { R:CCTTCTGTGTTTCATGTGTGATACC }\end{array}$ & $(\mathrm{TG})_{5}$ & $150-165$ & 9 & 0.64 & 5 & 0.41 \\
\hline $581 \mathrm{c}$ & GU253072 & $\begin{array}{l}\text { F:CCAACACACCAATAAACAATACCAG } \\
\text { R:TTTTGCAGAGAGAAAAGCACAGAC }\end{array}$ & $(\mathrm{CA})_{4}$ & $149-150$ & 2 & 0.06 & 1 & 0.00 \\
\hline $611 \mathrm{a}$ & GU253085 & $\begin{array}{l}\text { F:TGAAGGTTGGAGAGGAGAGAAATG } \\
\text { R:TCCAATATCACATGTAACCAAGCC }\end{array}$ & $(\mathrm{GA})_{4}$ & $117-127$ & 3 & 0.31 & 2 & 0.27 \\
\hline $616 a$ & GU253089 & $\begin{array}{l}\text { F:TTCGAAACATATTCTGACATGACTCC } \\
\text { R:CCTTGGGCTTTTCTGTTTTCTATTC }\end{array}$ & $(\mathrm{CTTT})_{4}$ & $128-133$ & 3 & 0.36 & 2 & 0.16 \\
\hline $625 a$ & GU253092 & $\begin{array}{l}\text { F:TTGTGGTTCTAGCCTTTGCTTTTC } \\
\text { R:ACTTTACGGTTGCAATCTCATTCC }\end{array}$ & $(\mathrm{GA})_{4}$ & $95-133$ & 4 & 0.36 & 2 & 0.32 \\
\hline $631 \mathrm{a}$ & GU253095 & $\begin{array}{l}\text { F:TTGCGAGTATGTATTTCGGTTGTG } \\
\text { R:TCTCTGTACCATTTTCATTTGGGAG }\end{array}$ & $(\mathrm{ATT})_{4}$ & $151-158$ & 3 & 0.15 & 1 & 0.00 \\
\hline $658 \mathrm{a}$ & GU253101 & $\begin{array}{l}\text { F:TTTTCAGAGCGTTATCACTCAACG } \\
\text { R:CCCTAAGTAGGAGCACTCCTTTCC }\end{array}$ & $(\mathrm{CT})_{6}$ & $97-123$ & 12 & 0.71 & 4 & 0.36 \\
\hline $660 \mathrm{a}^{\mathrm{y}}$ & GU171391 & $\begin{array}{l}\text { F:TAAAGCACAAAGTGCACAATCACC } \\
\text { R:GTTTTGGTTTGGTTTGGTGAAGAG }\end{array}$ & $(\mathrm{TC})_{7}$ & $97-132$ & 15 & 0.85 & 12 & 0.80 \\
\hline $671 \mathrm{a}$ & GU253108 & $\begin{array}{l}\text { F:AAGACATTGGAATTGGATTGGTTG } \\
\text { R:CTGCCGCTCTATCCTACCTCATAC }\end{array}$ & $(\mathrm{AG})_{10}$ & $105-151$ & 19 & 0.89 & 14 & 0.86 \\
\hline $680 \mathrm{a}$ & GU253111 & $\begin{array}{l}\text { F:AAATTTAAAGACCCCATTGCCAAC } \\
\text { R:ACACTCCCACAAAACCTTCACTTC }\end{array}$ & $(\mathrm{GT})_{8}$ & $144-152$ & 5 & 0.48 & 2 & 0.06 \\
\hline $6 \mathrm{a}^{\mathrm{y}}$ & GU171386 & $\begin{array}{l}\text { F:TCCACTTTGTTGAAATTGTTCTACG } \\
\text { R:TCATCCAAGTAATCATCCTTGCTG }\end{array}$ & $(\mathrm{GA})_{10}$ & $146-170$ & 7 & 0.62 & 6 & 0.63 \\
\hline $707 \mathrm{a}^{\mathrm{y}}$ & GU253119 & $\begin{array}{l}\text { F:GTGAGAGAAGGGATAACAGCTTGC } \\
\text { R:ACGCCCACTCTCACTCACAAC }\end{array}$ & $(\mathrm{GAA})_{6}$ & $142-160$ & 7 & 0.68 & 7 & 0.68 \\
\hline $732 \mathrm{a}$ & GU253126 & $\begin{array}{l}\text { F:ATTTTAATGAAGAGGAGAGCACGC } \\
\text { R:TGGGTAAAAGCCTCTTCAGTGTTC }\end{array}$ & $(\mathrm{AGA})_{5}$ & 99-102 & 2 & 0.32 & 2 & 0.37 \\
\hline $762 \mathrm{a}$ & GU253134 & $\begin{array}{l}\text { F:TCTGTCTCACCTGCTTGCACTAAG } \\
\text { R:GGCTCAATCTCCAAGAAAATGAAG }\end{array}$ & $(\mathrm{TC})_{7}$ & 94-111 & 8 & 0.70 & 5 & 0.52 \\
\hline $780 \mathrm{~b}$ & GU253139 & $\begin{array}{l}\text { F:TAGAGCCCTATTCCCACTTGACAC } \\
\text { R:CTTTATGAATGGTTGTCTTGCTGG }\end{array}$ & $(\mathrm{AG})_{12}$ & $142-175$ & 15 & 0.84 & 13 & 0.87 \\
\hline $830 \mathrm{a}$ & GU253159 & $\begin{array}{l}\text { F:GAGAAGCTAGACCATCCTACTCCG } \\
\text { R:TACCCTCGGACAACAAAGAGAATC }\end{array}$ & $(\mathrm{TC})_{7}$ & $154-164$ & 6 & 0.36 & 5 & 0.28 \\
\hline $834 a$ & GU253160 & $\begin{array}{l}\text { F:TCACGTTTAGTTCCCTTTTCAACC } \\
\text { R:TACATTCTGCAATTGTGGCTGC }\end{array}$ & $(\text { TTTA })_{4}$ & $150-168$ & 6 & 0.42 & 1 & 0.00 \\
\hline $847 \mathrm{a}$ & GU253165 & $\begin{array}{l}\text { F:ATTGCTCACCGTTCTTTCTCTCTG } \\
\text { R:ATGGAGAAGGTCAATAAGCAAACG }\end{array}$ & $(\mathrm{TC})_{4}$ & $104-128$ & 3 & 0.28 & 1 & 0.00 \\
\hline $849 a$ & GU253166 & $\begin{array}{l}\text { F:TCCTTTGAGTGTACATAGGTTATGCC } \\
\text { R:TCTTCTCTTCACTCTCTCAAACATTCC }\end{array}$ & $(\text { TTTA })_{4}$ & $204-217$ & 3 & 0.15 & 2 & 0.03 \\
\hline $863 a$ & GU253172 & $\begin{array}{l}\text { F:TTTCTTTTCTTCCCTCTCCATTCC } \\
\text { R:AAGAAGGAAAATGGGAAAGAGGC }\end{array}$ & $(\mathrm{TCT})_{4}$ & $83-89$ & 4 & 0.22 & 2 & 0.03 \\
\hline $871 \mathrm{a}$ & GU253176 & $\begin{array}{l}\text { F:TTCTTAAGCTAAACGGTGCATTTTG } \\
\text { R:GATGAGGGTTGGTGTAGTGAGGAG }\end{array}$ & $(\mathrm{CTT})_{9}$ & $112-159$ & 8 & 0.76 & 5 & 0.70 \\
\hline
\end{tabular}


Table 2. Continued.

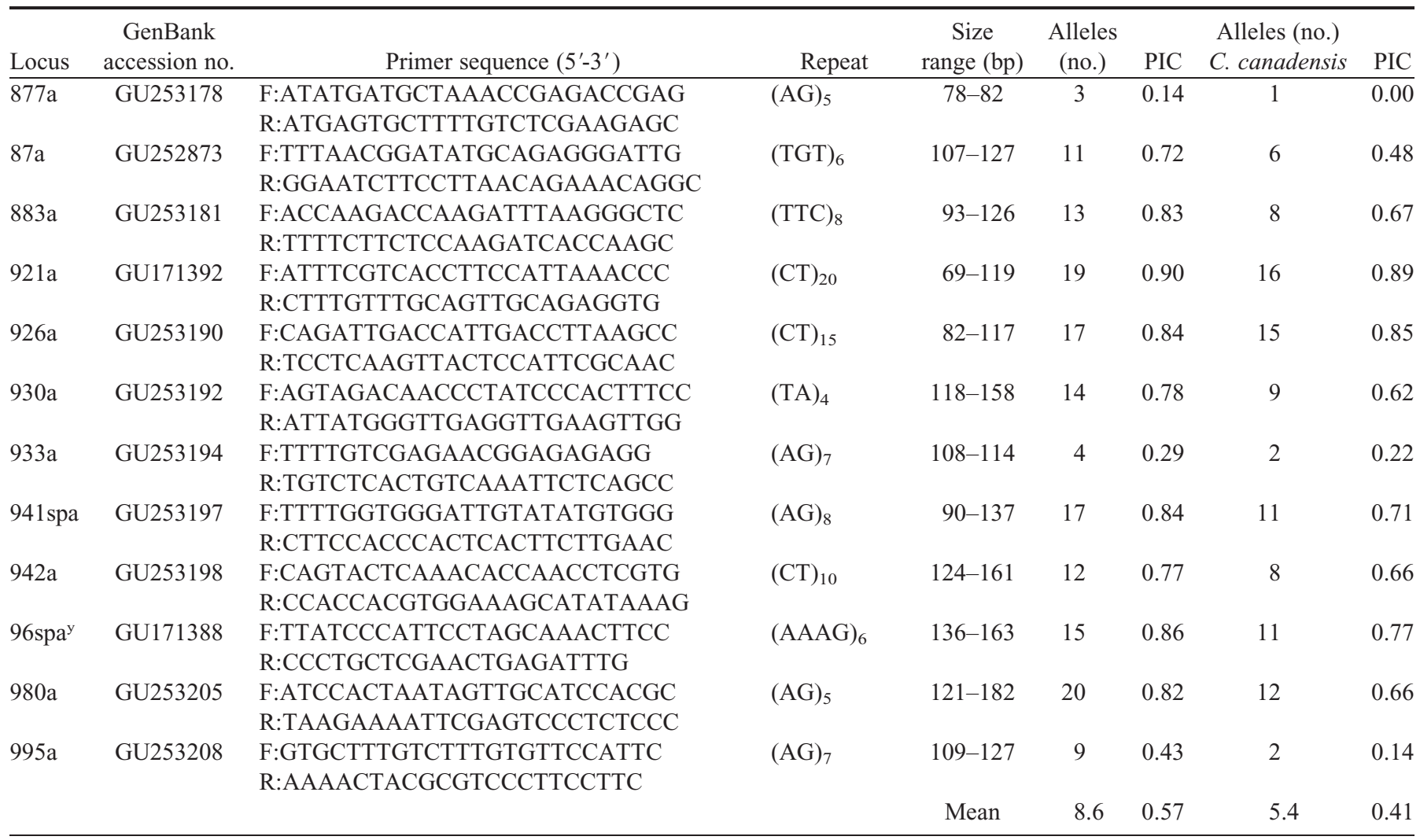

${ }^{\mathrm{z}}$ Shown for each locus are the GenBank accession number, forward and reverse primer sequence, repeat, number of alleles, size range (bp), and polymorphic information content (PIC). All values are based on the characterization of 50 samples from eight Cercis species and one sample of Bauhinia faberi.

${ }^{y}$ Loci that were previously reported in Rinehart et al. (2010).

(830a) failed to amplify and the number of alleles detected ranged from one to six. The number of alleles detected ranged from one to four for $C$. chingii and all loci amplified except for seven with 10 of these loci distinguishing the three accessions. Twenty of the 66 loci that amplified in C. gigantea were heterozygous. For the two samples of C. glabra tested, 67 loci amplified and 24 loci distinguished both samples; the number of alleles detected ranged from one to three. Only two loci $(847 \mathrm{a}$ and 871a) failed to amplify alleles in C. griffithii and 30 heterozygous loci were observed. C. occidentalis amplified at all but three loci (3a, 441a, 871a) with 24 heterozygous loci. The $C$. racemosa accession had the most (nine) loci that failed to amplify and of the amplified loci, only five were heterozygous. In the C. siliquastrum samples, one to four alleles were detected and all but two loci (164a and 268a) amplified with 12 loci needed to distinguish the samples. For all samples, the number of genotypes ranged from two $(581 \mathrm{c})$ to 34 (921a) and 15 loci distinguished 20 or more genotypes. The percent of cross-species transfer within Cercis was extremely high; 55 loci amplified across all the species and only two loci amplified $50 \%$ or less of the species $(830 \mathrm{a}=25 \%$ and $871 \mathrm{a}=50 \%$ ).

GENETIC RELATIONSHIPS BASED ON SIMPLE SEQUENCE REPEATS. The dendrogram produced by UPGMA using Nei et al. (1983) genetic distance matrix revealed the genetic relationships among all the taxa sampled (Fig. 1). The UPGMA tree showed clear structure, although the support values for most branches were modest. Three main clusters can be inferred from the dendrogram. There is one large cluster with the North American species (C. canadensis, C. canadensis var. mexicana, C. canadensis var. texensis, and $C$. occidentalis) and $C$. siliquastrum native to the Mediterranean region. Interestingly, the central Asian species C. griffithii also clustered with the North American species. The Asian species grouped into two clusters; one included C. chinensis, C. gigantea, C. glabra, and C. racemosa; and the second cluster contained only accessions of $C$. chingii.

The UPGMA dendrogram based on the SSR data of 13 individual plants of Cercis was similar to the tree with all individuals with three major clusters (Fig. 2). The largest cluster contained all of the Cercis species that are not native to China, whereas the Chinese species comprised the other two clusters. In one of the clusters, four of the five Chinese species were present and only individuals of $C$. chingii were in the second cluster.

INTERNAL TRANSCRIBED SPACER SEQUENCING AND PHYLOGENETIC RELATIONSHIPS. The length of the ITS region for the Cercis species ranged from 701 to $704 \mathrm{bp}$ and was $699 \mathrm{bp}$ in B. faberi. Clones of each accession revealed no polymorphisms within the ITS region. High homology was observed among the Cercis taxa analyzed. Sequence alignment produced a data matrix of 718 nucleotide characters, 20 (2.8\%) of which were informative.

Parsimony analysis using these 20 characters and B. faberi as an outgroup in a heuristic search revealed seven most parsimonious trees. One of these trees (Fig. 3) showed two 
Table 3. Number of alleles per locus, number of genotypes, and percent of cross-species transfer of 68 Cercis canadensis simple sequence repeat (SSR) loci in Cercis.

\begin{tabular}{|c|c|c|c|c|c|c|c|c|c|c|c|c|c|}
\hline \multirow[b]{2}{*}{ Species } & \multicolumn{13}{|c|}{ Alleles per locus (no. of genotypes) } \\
\hline & $1006 \mathrm{a}$ & $1057 \mathrm{a}$ & $1240 \mathrm{~b}$ & $127 \mathrm{spa}$ & $136 \mathrm{a}$ & $157 \mathrm{a}$ & $164 \mathrm{a}$ & $165 \mathrm{a}$ & $168 \mathrm{a}$ & $173 a$ & $176 \mathrm{a}$ & $177 \mathrm{~b}$ & $178 \mathrm{a}$ \\
\hline Bauhinia faberi (1) & - & 1 & - & - & - & - & - & - & - & 1 & - & - & - \\
\hline Cercis canadensis (26) & $2(2)$ & $9(15)$ & $3(3)$ & $4(6)$ & $2(2)$ & $1(1)$ & $5(6)$ & $5(6)$ & $2(3)$ & $2(3)$ & $3(4)$ & $2(3)$ & $4(4)$ \\
\hline $\begin{array}{l}\text { C. canadensis var. } \\
\text { mexicana (1) }\end{array}$ & 1 & 2 & 1 & 2 & 1 & 1 & 2 & - & - & 1 & - & 1 & 1 \\
\hline $\begin{array}{l}\text { C. canadensis var. } \\
\text { texensis ( } 3 \text { ) }\end{array}$ & $2(2)$ & $4(3)$ & $2(2)$ & $4(3)$ & $4(3)$ & $1(1)$ & $2(2)$ & $1(1)$ & $3(2)$ & $2(2)$ & $2(3)$ & $2(2)$ & $2(3)$ \\
\hline C. chinensis (8) & $4(4)$ & $2(2)$ & $2(2)$ & $2(2)$ & $2(2)$ & 1 & $2(2)$ & $2(2)$ & $2(2)$ & 1 & 1 & $2(2)$ & 1 \\
\hline C. chingii (3) & 1 & $3(3)$ & $2(1)$ & 1 & $3(2)$ & $2(2)$ & $3(3)$ & $2(2)$ & $2(2)$ & 1 & $2(2)$ & $2(3)$ & $2(3)$ \\
\hline C. gigantea (1) & 1 & 2 & 2 & 2 & 1 & 2 & - & 1 & 1 & 1 & 1 & 2 & 1 \\
\hline C. glabra (2) & $2(2)$ & 1 & 1 & $3(2)$ & $2(2)$ & 1 & $2(2)$ & $3(2)$ & 1 & 1 & 1 & $2(2)$ & 1 \\
\hline C. griffithii (1) & 1 & 2 & 2 & 2 & 1 & 1 & 1 & 2 & 2 & 1 & 1 & 2 & 1 \\
\hline C. occidentalis (1) & 1 & 2 & 1 & 1 & 1 & 2 & 1 & 2 & 1 & 1 & 1 & 1 & 1 \\
\hline C. racemosa (1) & 1 & 1 & 1 & 1 & 1 & 2 & 1 & 2 & - & 1 & 1 & 1 & 1 \\
\hline C. siliquastrum (3) & 1 & $2(2)$ & $2(2)$ & $4(3)$ & 1 & $2(1)$ & - & $3(2)$ & 1 & 1 & 1 & 1 & 1 \\
\hline Total alleles & $6(8)$ & $11(22)$ & $6(7)$ & $10(13)$ & $8(9)$ & $3(3)$ & $8(11)$ & $7(14)$ & $4(5)$ & $4(5)$ & $5(6)$ & $8(10)$ & $6(10)$ \\
\hline $\begin{array}{l}\text { Percent transfer } \\
\text { Cercis sp. }\end{array}$ & 100 & 100 & 100 & 100 & 100 & 100 & 75 & 100 & 88 & 100 & 100 & 100 & 100 \\
\hline
\end{tabular}

\begin{tabular}{|c|c|c|c|c|c|c|c|c|c|c|c|c|c|}
\hline \multirow[b]{2}{*}{ Species } & \multicolumn{13}{|c|}{ Alleles per locus (no. of genotypes) } \\
\hline & $180 \mathrm{a}$ & $199 a$ & $220 \mathrm{a}$ & $221 \mathrm{a}$ & $229 a$ & $254 \mathrm{a}$ & $268 \mathrm{a}$ & $272 a$ & $287 b$ & $324 a$ & $37 \mathrm{a}$ & $386 \mathrm{~b}$ & $3 a$ \\
\hline Bauhinia faberi (1) & - & - & - & - & - & 1 & - & - & - & 2 & - & 1 & - \\
\hline Cercis canadensis (26) & $6(7)$ & $3(4)$ & $3(4)$ & $3(4)$ & $2(3)$ & $5(8)$ & $2(3)$ & $2(2)$ & $3(3)$ & $7(11)$ & $8(13)$ & $2(3)$ & $10(13)$ \\
\hline $\begin{array}{l}\text { C. canadensis var. } \\
\text { mexicana (1) }\end{array}$ & 1 & 2 & 2 & 1 & 1 & 1 & 1 & 1 & 1 & 1 & 2 & 1 & - \\
\hline $\begin{array}{l}\text { C. canadensis var. } \\
\text { texensis (3) }\end{array}$ & $3(3)$ & $1(1)$ & $2(2)$ & $4(3)$ & 1 & 1 & $2(2)$ & 1 & $2(2)$ & $4(3)$ & 1 & $3(2)$ & - \\
\hline C. chinensis (8) & 1 & $2(2)$ & $2(2)$ & 1 & 1 & $5(6)$ & 1 & $2(3)$ & $2(2)$ & 1 & $6(5)$ & 1 & $3(3)$ \\
\hline C. chingii (3) & 1 & - & 1 & 1 & $2(2)$ & $2(1)$ & $2(2)$ & $2(2)$ & - & 1 & 1 & 1 & - \\
\hline C. gigantea (1) & 2 & 1 & 1 & 1 & 1 & 1 & 1 & 1 & 1 & 1 & 1 & 1 & 2 \\
\hline C. glabra (2) & $2(2)$ & 1 & $2(2)$ & 1 & 1 & $3(2)$ & 1 & 1 & 1 & 1 & $2(2)$ & 1 & $3(2)$ \\
\hline C. griffithii (1) & 1 & 1 & 2 & 2 & 1 & 1 & 1 & 1 & 1 & 2 & 1 & 2 & 2 \\
\hline C. occidentalis (1) & 1 & 2 & 1 & 1 & 2 & 1 & 2 & 2 & 2 & 1 & 1 & 1 & - \\
\hline C. racemosa (1) & 1 & 1 & 1 & 1 & 1 & 1 & - & 1 & 1 & 1 & 1 & 1 & - \\
\hline C. siliquastrum (3) & 1 & $2(2)$ & $2(2)$ & $2(2)$ & $2(2)$ & $4(3)$ & $2(2)$ & $2(2)$ & 1 & $2(2)$ & $2(2)$ & 1 & 1 \\
\hline Total alleles & $7(12)$ & $8(8)$ & $5(8)$ & $8(10)$ & $8(9)$ & $12(20)$ & $8(10)$ & $2(3)$ & $7(8)$ & 12 (19) & $16(23)$ & $5(6)$ & $13(18)$ \\
\hline $\begin{array}{l}\text { Percent cross-species } \\
\text { transfer }\end{array}$ & 100 & 88 & 100 & 100 & 100 & 100 & 88 & 100 & 88 & 100 & 100 & 100 & 63 \\
\hline
\end{tabular}

Alleles per locus (no. of genotypes)

\begin{tabular}{|c|c|c|c|c|c|c|c|c|c|c|c|c|c|}
\hline Species & $402 a$ & $416 \mathrm{a}$ & $441 \mathrm{a}$ & $461 \mathrm{a}$ & $475 \mathrm{a}$ & $508 \mathrm{a}$ & $519 \mathrm{a}$ & $53 a$ & $563 a$ & $579 a$ & $581 \mathrm{c}$ & $611 \mathrm{a}$ & $616 \mathrm{a}$ \\
\hline Bauhinia faberi (1) & 1 & - & - & - & - & - & - & - & 1 & - & - & - & - \\
\hline $\begin{array}{l}\text { C. canadensis var. } \\
\text { mexicana }(1)\end{array}$ & 1 & 1 & 1 & 1 & 1 & 1 & 2 & 1 & 2 & - & 1 & 1 & 1 \\
\hline $\begin{array}{l}\text { C. canadensis var. } \\
\text { texensis (3) }\end{array}$ & $2(2)$ & $2(2)$ & 1 & $3(3)$ & 1 & $2(1)$ & $2(1)$ & 1 & $3(2)$ & $3(3)$ & 1 & 1 & $2(2)$ \\
\hline C. chinensis (8) & 1 & 1 & 1 & $2(2)$ & 1 & 1 & 1 & $3(4)$ & $2(2)$ & 1 & 1 & 1 & 1 \\
\hline C. gigantea (1) & 1 & 1 & 1 & 1 & 1 & 1 & 2 & 2 & 1 & 2 & 1 & 1 & 1 \\
\hline C. glabra (2) & 1 & 1 & 1 & $2(2)$ & 1 & 1 & 1 & $2(2)$ & 1 & 1 & 1 & 1 & $2(2)$ \\
\hline C. griffithii (1) & 1 & 1 & 1 & 2 & 2 & 1 & 1 & 2 & 2 & 2 & 1 & 2 & 1 \\
\hline C. occidentalis (1) & 1 & 1 & - & 2 & 2 & 1 & 1 & 2 & 1 & 1 & 1 & 1 & 1 \\
\hline C. racemosa (1) & 1 & 1 & - & 1 & 1 & 1 & 2 & 1 & 1 & 1 & 1 & 1 & 1 \\
\hline C. siliquastrum (3) & $3(3)$ & $3(2)$ & $3(3)$ & $2(2)$ & $2(2)$ & 1 & $3(3)$ & 1 & $3(3)$ & $3(3)$ & 1 & 1 & 1 \\
\hline
\end{tabular}


Table 3. Continued.

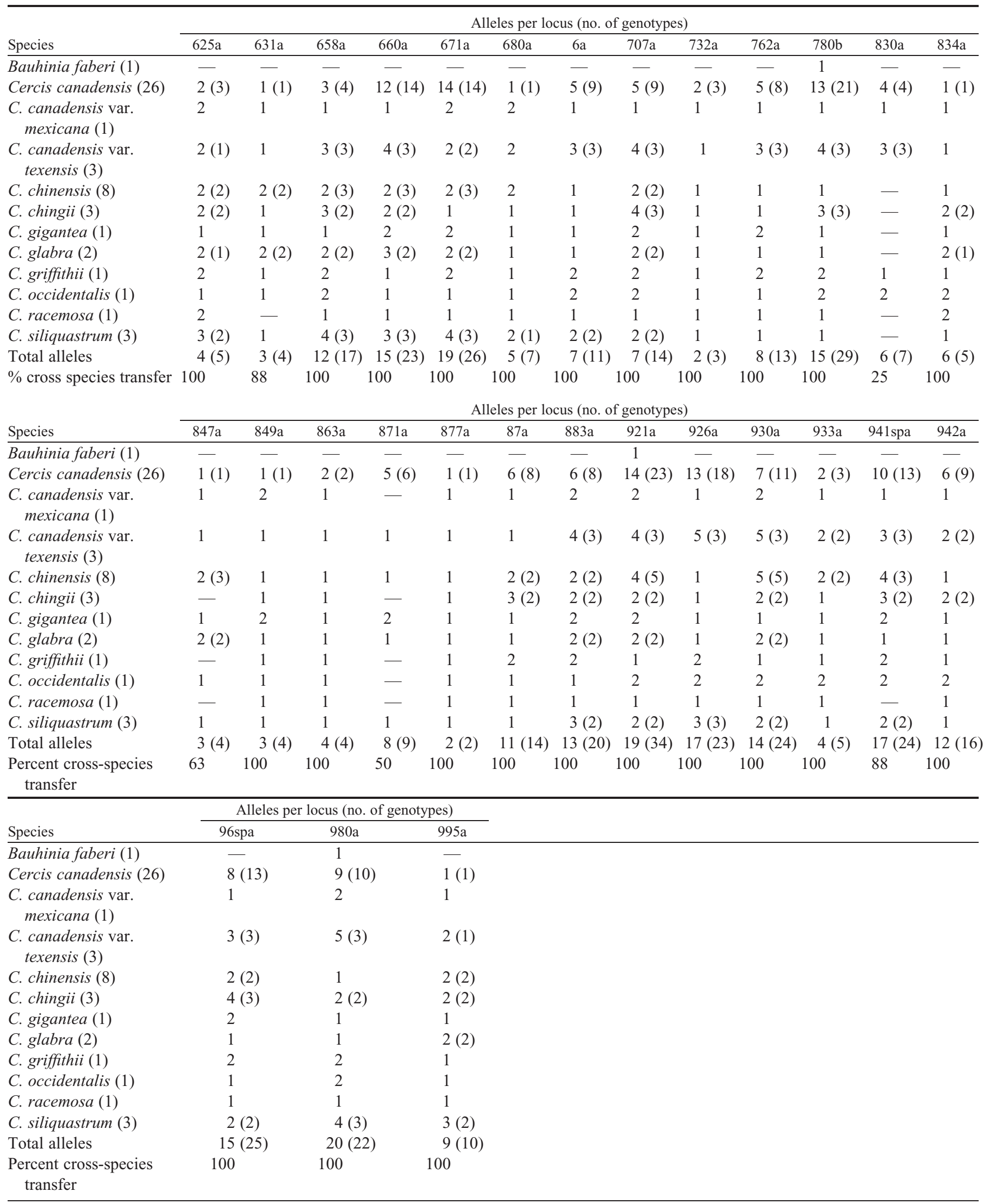




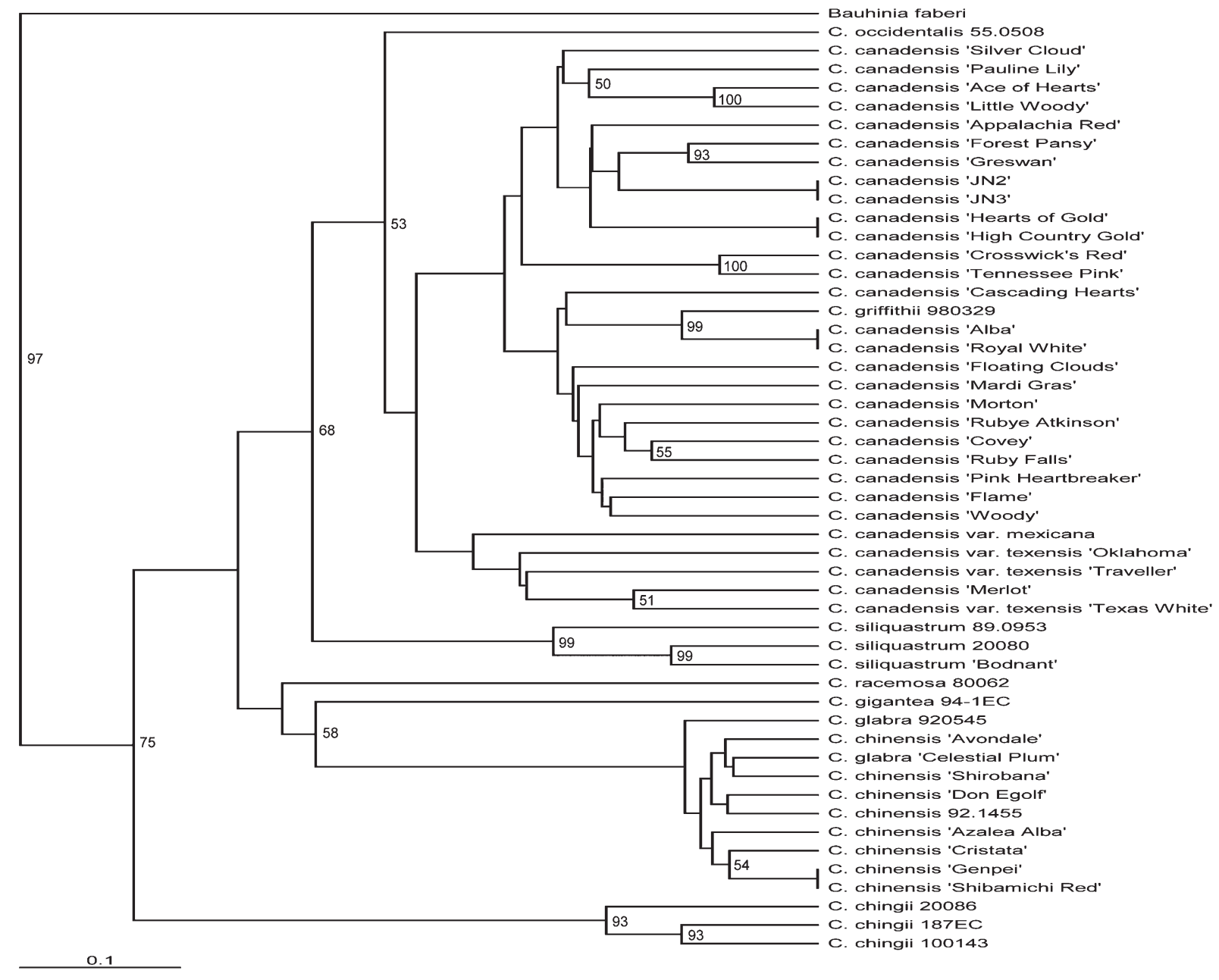

Fig. 1. Unweighted pair group method with arithmetic mean (UPGMA) tree showing the relationships of 50 Cercis cultivars and accessions and a related genera (Bauhinia faberi) as determined by the genetic distance matrix (Da) of Nei et al. (1983) of 68 simple sequence repeat (SSR) loci. Bootstrap analysis was performed with 500 replications and values greater than 50 are indicated to the right of the node. Data were analyzed with Populations 1.2.32 (Langella, 2002) and the UPGMA tree was visualized in the program TreeView (Page, 1996).

branches with bootstrap values having modest (56) and robust (99) support. A single clade comprised of the three $C$. chingii accessions was identified with high bootstrap support that was basal to all of the remaining Cercis taxa. This latter clade was further subdivided into two subclades. The more basal section included the Chinese species $C$. chinensis, C. glabra, C. gigantea, and C. racemosa. The second subclade included the remaining taxa and $C$. canadensis 'Ace of Hearts' and C. canadensis var. texensis 'Oklahoma'. C. griffithii aggregated into a separate group from $C$. canadensis var. mexicana, $C$. occidentalis, and $C$. siliquastrum.

\section{Discussion}

SimPLE SEQUenCe REPEAT TRANSFERABILITY. Transferability across different species was assessed with $C$. canadensis SSRs in eight Cercis species. From 68 loci there were potentially 3400 PCR amplifications and 3284 (96.6\%) amplified. Modification of PCR protocols is commonplace and Rossetto (2001) reviewed transferability of SSRs in plants and reported that $65 \%$ of the protocols were modified. In a review of 64 primer notes published in Molecular Ecology and Molecular Ecology Notes (1997-2006) in which cross-species transfer of SSRs was reported, Barbará et al. (2007) compiled the percent of cross- species amplification and polymorphism based on the number of cross-species encounters for 14 published reports on eudicots. In 127 encounters, the mean amplification was $71 \%( \pm 2)$ and the median was $80 \%$. Of 49 potential polymorphic encounters, the mean was $48 \%( \pm 4)$ and the median was $46 \%$. Our results for percent cross-species amplification (95\% average for 68 loci) are much higher and our rate of $37.1 \%$ cross-species polymorphisms was slightly lower.

GENETIC RELATIONSHIPS BASED ON SIMPLE SEQUENCE REPEATS AND INTERNAL TRANSCRIBED SPACER SEQUENCING. There are currently 11 recognized Cercis species (Dezhao et al., 2010) and species delimitation has been difficult using morphological characters, which are largely quantitative, continuous, and are of limited use for phylogenetic resolution (Davis et al., 2002; Zhang, 1994, 1999). Because of lack of morphological delineators, DNA sequence data of the nuclear ribosomal ITS region (Coşkun and Parks, 2009a; Davis et al., 2002; Zhang, 1994, 1999) and the chloroplast gene $n d h F$ (Coskun and Parks 2009b; Davis et al., 2002) and combination of plastid (ndhF, rpoB-trnC, trnT-trnD, and $\operatorname{trnS}$-trn $G$ ) and nuclear (ITS) regions (Fritsch and Cruz, 2012) have been used to reconstruct phylogenies of Cercis with varying degrees of success. One of the main constraints of sequence polymorphism markers is limited sequence variation. We applied 


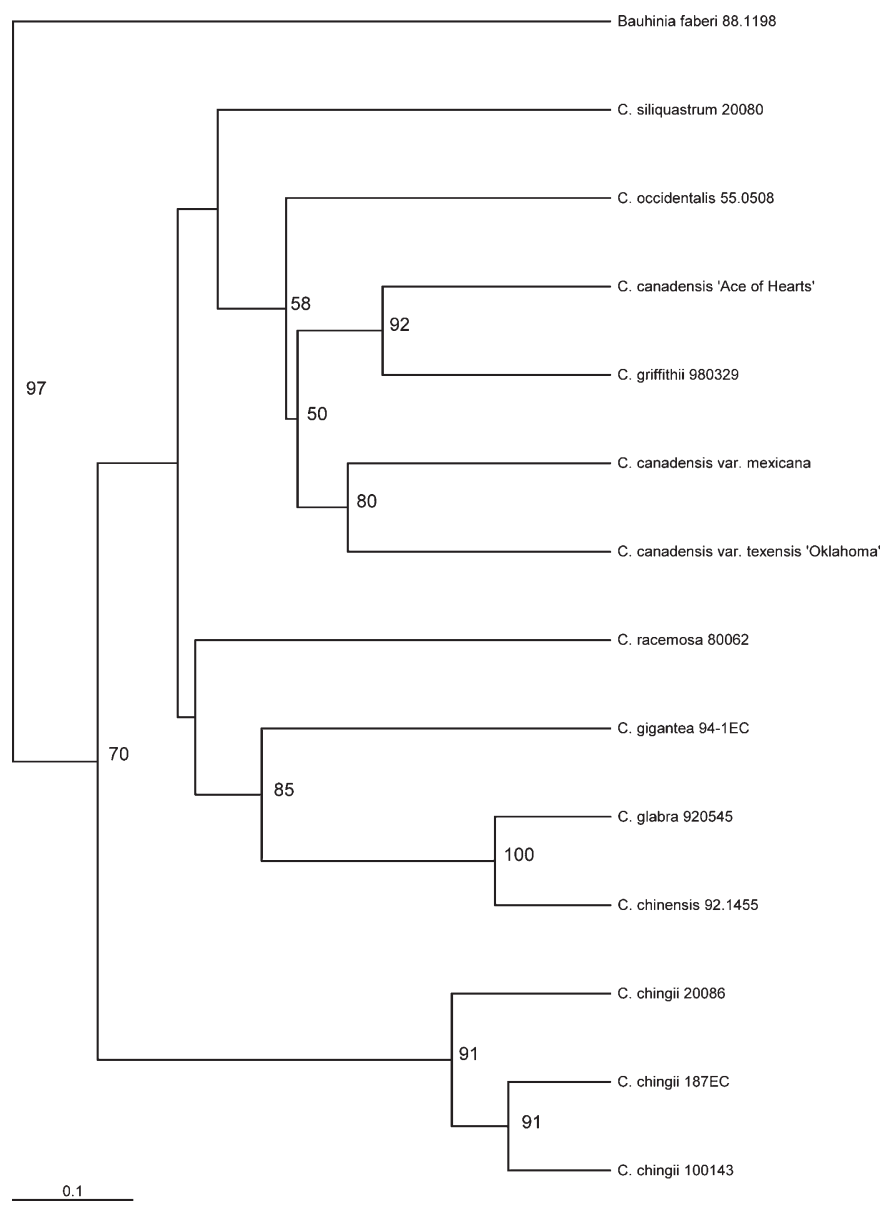

Fig. 2. Unweighted pair group method with arithmetic mean (UPGMA) tree showing the relationships of eight recognized Cercis species and a related genus (Bauhinia faberi) as determined by the genetic distance matrix (Da) of Nei et al. (1983) based on 68 simple sequence repeat (SSR) loci. Bootstrap analysis was performed with 500 replications and values greater than 50 are indicated to the right of the node. Data were analyzed with Populations 1.2.32 (Langella, 2002) and the UPGMA tree was visualized in the program TreeView (Page, 1996).

SSR markers as an alternative because SSRs are codominant, widely distributed throughout the genome, highly polymorphic, reproducible, and are useful in studying close genetic relationships. Additionally, the evolution of SSRs generates polymorphisms at a faster rate and can potentially be applied to phylogentic studies. SSRs have been used for over 20 years to study the genetic relationships of plants but have rarely been used in phylogenetic inference (Calonje et al., 2009). Two reasons for this are the large numbers of different SSRs that are needed to provide a wellresolved phylogeny are usually unavailable (Takezaki and Nei, 1996), and to date, most studies have generally used only 10 or less loci (Calonje et al., 2009). With the 68 SSRs that we have available for C. canadensis, we feel that we can provide insight into phylogenetic relationships among Cercis species and overcome some of the stated limitations.

The SSR data showed clear structure based on the current taxonomic classification of species within the genus, although support values for most branches were modest. Generally, the bootstrap values were higher when more taxa were included in the analysis and the North American and western Eurasian species formed a clade. Regardless of the samples included, the UPGMA analysis revealed three clusters, and all of the Chinese

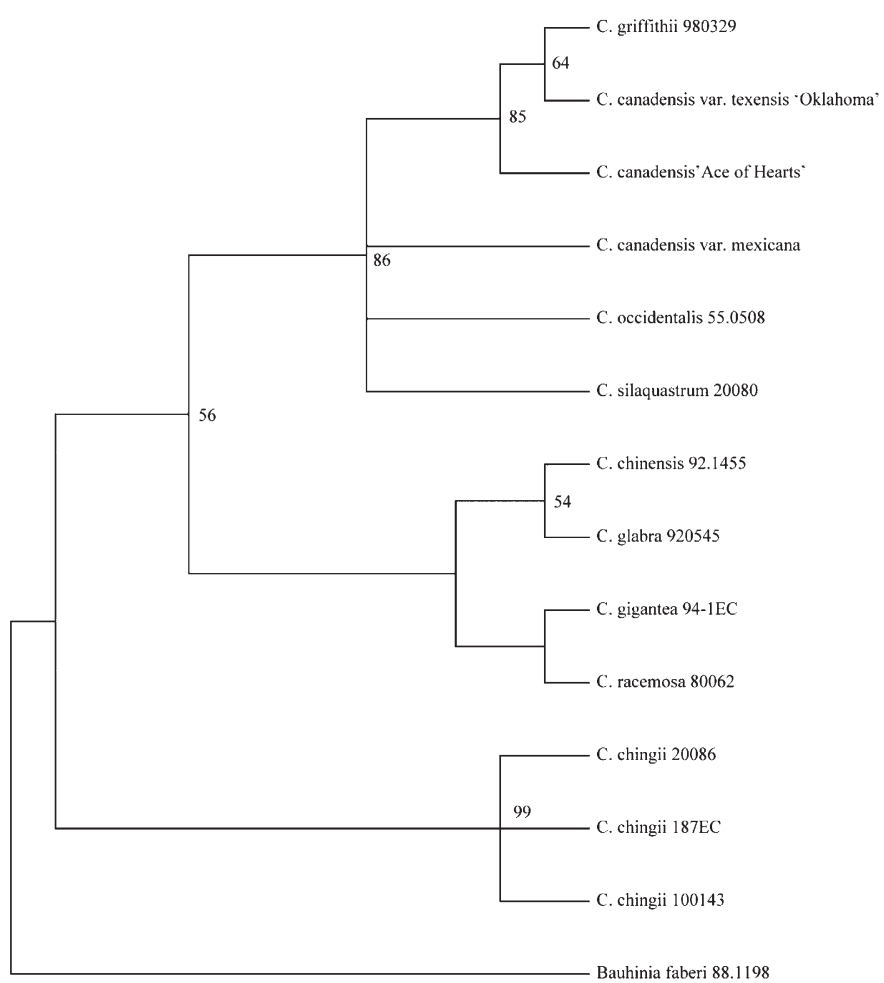

Fig. 3. One of the seven most parsimonius trees generated by parsimony analysis of internal transcribed spacer (ITS) region sequences showing the relationships of eight recognized Cercis species and a related genera (Bauhinia faberi). Bootstrap analysis was performed with 1000 replications and values greater than 50 are indicated to the right of the node. Data were analyzed with PAUP* (Sinauer Associates, Sunderland, MA) and trees were visualized in the program TreeView (Page, 1996).

species grouped separately from the other taxa. Additionally, both analyses grouped $C$. chingii alone with strong support and there was no support for clustering of C. racemosa with C. chinensis, C. gigantea, and C. glabra. The third cluster, as expected, grouped the North America species together, but unexpectedly grouped $C$. griffithii, a Eurasian species, with these species too. The remaining species, C. siliquastrum, grouped in the UPGMA analysis with support in the cluster that included all individuals and without support (bootstrap = 48) when only 13 accessions were used in the analysis.

The widely cultivated $C$. canadensis and $C$. chinensis comprised the majority of the samples analyzed and the bootstrap values greater than 50 for the cultivars of $C$. canadensis are in agreement with their pedigree. For example, 'Ace of Hearts' and 'Little Woody' clustered with $100 \%$ support in the $C$. canadensis group, and both of these dwarf cultivars were selected from a group of 300 to 400 open-pollinated seedlings (Fantz and Woody, 2005). Both 'Forest Pansy' and 'Greswan' are red-leaved selections that, based on our study, were potentially derived from the same germplasm. The other two cultivars with burgundy-colored foliage, 'Merlot' and 'Ruby Falls', clustered separately from 'Forest Pansy' and 'Greswan' as expected based on their diverse pedigrees. 'Ruby Falls' is derived from the controlled cross of 'Covey' $\times$ 'Forest Pansy' and has a weeping growth habit similar to 'Covey' and the purple leaf color of 'Forest Pansy', whereas 'Merlot' originated from a $F_{2}$ family resulting from the cross of 'Texas White' $\times$ 'Forest Pansy' (Werner and Snelling, 2010). 
The patent protected 'JN2' and 'JN3' were identical at 68 loci, which is congruent with ' $\mathrm{JN} 3$ ' being discovered as a bud sport of 'JN2'. Both cultivars exhibit new growth that is golden orange that matures to lime green, but ' $\mathrm{JN} 3$ ' has a wavy, rugose, dark green leaf margin (Jackson and Jackson, 2011). 'Hearts of Gold' (PP17,740; Roethling, 2007) and 'High Country Gold' (plant patent applied for) were also identical at 68 loci and also appear to be phenotypically very similar. Identical SSR data for the two cultivars could be explained if 'High Country Gold' was a sport of 'Hearts of Gold', which was discovered in 2002 as an isolated seedling of $C$. canadensis and awarded a U.S. Plant Patent in 2007, although the origin of 'High Country Gold' is undocumented. Because they are codominant and widespread in the nuclear genome, SSR markers can reliably verify parentage. However, SSR markers typically cannot distinguish between clonal copies or bud sport mutations, both of which should share identical genomes at all or nearly all loci. For example, in cases in which two accessions that are labeled differently and expected heterozygosity of the SSR markers is very low or zero, it is likely that one was derived from the other as a sport or clonal mutant rather than a progeny or sibling.

Interestingly, $C$. griffithii, which is native to central Asia, clustered with 'Alba' and 'Royal White'. We were puzzled by this relationship and suspected that the $C$. griffithii accession was potentially misidentified. Mislabeling was confirmed by the Curator of Collections at the JC Raulston Arboretum at North Carolina State University, Raleigh (M. Weathington, personal communication) who provided the original tissue included in the analyses. With the high bootstrap support of this sample clustering with $C$. canadensis in both UPGMA analyses, we speculate that the mislabeled $C$. griffithii is an accession of $C$. canadensis of an unknown origin. The only two white-flowered cultivars, Alba and Royal White, were identical at all loci tested. 'Alba' is the most likely applied moniker to any of the white-flowered forms available in the nursery industry and has been stated to be less coldhardy than the seedling selection 'Royal White', whose flowers are larger and open slightly earlier (Dirr, 1998). Careful morphological comparisons are needed to determine if the 'Alba' and 'Royal White' samples in our study are the same clone sold under two names or sports with consistent phenotypic differences.

Both analyses of the SSR data revealed bootstrap support for C. gigantea as a distinct species from C. glabra, but support was lacking in our ITS data. Based on these results, we make the same suggestion as Dezhao et al. (2010) that further investigation is recommended to determine if $C$. gigantea is a distinct species. The other unresolved relationship involves the unsupported clustering of $C$. glabra 'Celestial Plum' with the $C$. chinensis accession and cultivars. 'Celestial Plum' was originally named and is still found in the nursery industry as C. yunnanensis $\mathrm{Hu} \&$ Cheng (Weathington, 2009), which is synonymous with C. glabra (Dezhao et al., 2010). Both C. glabra and C. chinensis occur in the same Chinese province (Dezhao et al., 2010). Furthermore, 'Celestial Plum' was a seedling selected from the Yunnan Province that was released by the J.C. Raulston Arboretum with noted similarity to $C$. chinensis (Raulston, 1995). It could potentially be a cultivar of $C$. chinensis, because the other accession of C. glabra clustered alone within this group.

Parsimony analysis of the ITS sequences revealed support for three groups. Once again, accessions of $C$. chingii grouped separately from all the other species with high support and the remaining Chinese species formed a group separate from $C$. canadensis, C. griffithii, C. occidentalis, and C. siliquastrum.
Because this is the first evaluation of genetic relationships among cultivars and accessions of Cercis species based on SSRs, there are no studies to compare with our results. However, multiple studies have used ITS sequencing to infer the phylogenetic relationships within Cercis (Coşkun and Parks, 2009a; Davis et al., 2002; Fritsch and Cruz, 2012; Hao et al., 2001). Using maximum parsimony analysis of eight Cercis species, Hao et al. (2001) found consistent grouping of the North American species (C. canadensis and $C$. occidentalis) with $C$. siliquastrum with moderate support that nested within the Chinese species. These authors also suggested that further phylogenetic analyses of Cercis were needed using additional data as a result of the poor resolution of their strict consensus tree. The phylogenetic analysis of the ITS region of Davis et al. (2002) resolved C. chingii as the sister species to all other Cercis species. This analysis also showed bootstrap support for a clade of the $C$. canadensis accessions, a clade each for the two accessions of $C$. occidentalis and $C$. siliquastrum, a clade with $C$. chinensis and $C$. glabra, and a clade of the two accessions of $C$. racemosa. The discussion of phylogenetic relationships was confined to divergence between the North American (C. canadensis and $C$. occidentalis) and western Eurasian ( $C$. siliquastrum) species. They estimated that the divergence between these species occurred 6 to 32 million years, which they found inconsistent with current geological and climatic interpretations. Additional fossil and molecular data are needed to resolve phylogenetic relationships in this group. Coskun and Parks (2009a) found bootstrap support for a clade containing C. californica Torr. Ex Benth. (presumed to be C. occidentalis), the C. canadensis botanical varieties, $C$. griffithii, and C. siliquastrum; a clade containing $C$. glabra, C. racemosa, and C. yunnanensis; and a clade of $C$. chuniana and $C$. occidentalis. Finally, Fritsch and Cruz (2012) reported trees that were mostly unresolved with generally low support of clades and these results are similar to the previous studies.

Our phylogenetic relationships for Cercis are more consistent with those of Davis et al. (2002) and Hao et al. (2001) than those of Coşkun and Parks (2009a) and Fritsch and Cruz (2012) with regard to the North American and western Eurasian species. One exception to this is the well-supported relationship between $C$. canadensis and $C$. griffithii that we found in our ITS and SSR analyses. Because they used the same source of $C$. griffithii for their analysis, Coşkun and Parks (2009a) found support for this same relationship. Fritsch and Cruz (2012) also reported similar results for this relationship, although their $C$. griffithii accession was different from what was used in our study and by Coşun and Parks (2009a). However, based on morphological and molecular traits, we are confident that our sample of $C$. griffithii is mislabeled and is actually $C$. canadensis. In all of our analyses, $C$. chingii resolved as a sister clade to the other species, in agreement with Davis et al. (2002) and Fritsch and Cruz (2012), but not with other studies. This discrepancy could be the result of the differing number of taxa used in each study. Zwickl and Hillis (2002) examined five aspects of study design for phylogenetic inference and found that for all simulation analyses conducted, increased taxon sampling is one of the most important factors leading to increased phylogenetic accuracy.

The North American species of Cercis are proposed to be monophyletic (Hopkins, 1942; Isley, 1975; McVaugh, 1952), and our ITS data are in agreement with this hypothesis. However, our SSR data do not support this hypothesis but rather are in agreement with the ITS data of Hao et al. (2001). Davis et al. (2002) using ITS data also found that the North American species 
are not monophyletic but interestingly found that $C$. canadensis is more closely related to $C$. siliquastrum from western Eurasia than to $C$. occidentails from western North America. They suggested that more investigation is needed to confirm these results because the bootstrap support for this topology was weak. All of our analyses (SSRs and ITS) are in agreement with the findings of Davis et al. (2002), Fritsch and Cruz (2012), and Hao et al. (2001) in regard to $C$. canadensis being a distinct species from $C$. occidentalis. Because of the varying number of species and taxa used and the relatively low bootstrap support found in our study and the previous phylogenetic studies of Cercis based on ITS sequences (Coşkun and Parks 2009a; Davis et al., 2002; Fritsch and Cruz, 2012; Hao et al., 2001), we suggest that the relationships be evaluated with additional data, including more accessions and more or different markers. More specifically, considering the most resolved and published phylogenetic estimates of Cercis based on a combined ITS-plastid analysis reported by Fritsch and Cruz (2012), further studies are needed for resolution of North American species.

IMPLICATIONS FOR CERCIS BREEDING AND GENETICS. Although our study did not provide complete resolution of all the genetic relationships within Cercis, the SSRs analyzed have broad application for the genus. The high rates of cross-species transfer of the loci provided in this study indicate that these markers are well suited for genome studies in Cercis. Additionally, the observed PIC values indicate the suitability of these markers for mapping studies involving $C$. canadensis and $C$. chinensis. The molecular markers presented in our study will allow breeders to be more efficient by using the information provided by the genetic relationships within the cultivars of $C$. canadensis and $C$. chinensis to design more efficient controlled crosses. The broad cross-species transfer of the SSRs also suggests the potential for performing wide hybridizations within Cercis to improve the traits of cultivated redbuds. For example, fungal canker, caused by Botryosphaeria ribis Grossenbacher \& Duggar, is considered to be the most destructive disease of $C$. canadensis (Dirr, 1998) and could limit its use in landscapes (Pooler et al., 2002). Considering that the Asian redbud species are less susceptible to canker (Pooler et al., 2002), wide hybridization offers the potential to introduce resistance into $C$. canadensis.

In summary, we have documented the wide cross-species transfer of SSRs from C. canadensis, assessed the genetic relationships within Cercis, and provided guidance to which taxonomic level the SSR loci are useful for breeding and genetics of Cercis.

\section{Literature Cited}

Baldwin, B.G., C.S. Campbell, J.M. Porter, M.J. Sanderson, M.F. Woijciechowski, and M.J. Donoghue. 1995. Utility of nuclear ribosomal DNA internal transcribed spacer sequences in phylogenetic analyses of angiosperms. Ann. Mo. Bot. Gard. 82:247277.

Barbará, T., C. Palma-Silva, G.M. Paggi, F. Bered, M.F. Fay, and C. Lexer. 2007. Cross-species transfer of nuclear microsatellite markers: Potential and limitations. Mol. Ecol. 16:3759-3767.

Brownstein, M.J., J.D. Carpten, and J.R. Smith. 1996. Modulation of non-templated nucleotide addition by Taq DNA polymerase: Primer modifications that facilitate genotyping. Biotechniques 20:10041006, 1008-1010.

Calonje, M., S. Martin-Bravo, C. Dobes, W. Gong, I. Jordon-Thaden, C. Kiefer, M. Kiefer, J. Paule, R. Schmickl, and M.A. Koch. 2009. Non-coding nuclear DNA markers in phylogenetic reconstruction. Plant Syst. Evol. 282:257-280.
Cosskun, F. and C.R. Parks. 2009a. A molecular phylogenetic study of red buds (Cercis L., Fabaceae) based on ITS nrDNA sequences. Pak. J. Bot. 41:1577-1586.

Coşkun, F. and C.R. Parks. 2009b. A molecular phylogeny of Cercis L. (Fabaceae) using the chloroplast trnL-F DNA sequences. Pak. J. Bot. 41:1587-1592.

Davis, C.D., P.W. Fritsch, J. Li, and M.J. Donoghue. 2002. Phylogeny and biogeography of Cercis (Fabaceae): Evidence from nuclear and ribosomal ITS and chloroplast $n d h F$ sequence data. Syst. Bot. 27:289-302.

Dezhao, D., Z. Dianxiang, S.S. Larsen, and M.A. Vincent. 2010. Cercis Linnaeus, Sp. P1. 1:374. 1753. Flora China 10:5-6.

Dirr, M.A. 1998. Manual of woody landscape plants: Their identification, ornamental characteristics, culture, propagation, and uses. Stipes Publishing, Champaign, IL.

Fantz, P.R. and P. Woody. 2005. 'Ace of Hearts' and 'Little Woody' redbuds. HortScience 40:2209-2210.

Fritsch, P.W. and B.C. Cruz. 2012. Phylogeny of Cercis based on DNA sequences of nuclear ITS and four plastid regions: Implications for transatlantic historical biogeography. Mol. Phylogenet. Evol. 62:816-825.

Fritsch, P.W., A.M. Schiller, and K.W. Larson. 2009. Taxonomic implications of morphological variation in Cercis canadensis (Fabeae) from Mexico and adjacent parts of Texas. Syst. Bot. 34:510-520

Hao, G., D.X. Zhang, L.X. Guo, Y.F. Deng, and X.Y. Wen. 2001. A phylogenetic and biogeographic study of Cercis (Leguminosae). Acta Bot. Sin. 43:1275-1278.

Hopkins, M. 1942. Cercis in North America. Rhodora 44:192-211.

Isley, D. 1975. Leguminosae of the United States: II. Subfamily Caesalpinioideae. Memoirs New York Bot. Garden 25:1-228.

Jackson, R. and C. Jackson. 2011. Eastern redbud tree named 'JN3'. U.S. Plant Patent US 2011/0023196 P1. U.S. Patent and Trademark Office, Washington, DC.

Kalinowski, S.T., M.L. Taper, and T.C. Marshall. 2007. Revising how the computer program CERVUS accommodates genotyping error increases success in paternity assignment. Mol. Ecol. 16:10991106.

Langella, O. 2002. Populations, a free populations genetics software (version 1.2.31). 1 Dec. 2011. < http://bioinformatics.org/ tryphon/ populations/>.

Li, H.L. 1944. Taxonomy and distribution of the genus Cercis in China. Bull. Torrey Bot. Club 71:419-425.

McVaugh, R. 1952. Suggested phylogeny of Prunus serotina and other wide-ranging phylads in North America. Brittonia 7:317-346.

Nei, M., F. Tajima, and Y. Tateno. 1983. Accuracy of estimated phylogenetic trees from molecular data. J. Mol. Evol. 19:153-170.

Page, R.D. 1996. TreeView: An application to display phylogenetic trees on personal computers. Comput. Appl. Biosci. 124:357-358.

Pooler, M.R., K.A. Jacobs, and K. Kramer. 2002. Differential resistance to Botryosphaeria ribis among Cercis taxa. Plant Dis. 86:880-882.

Raulston, J.C. 1995. Friends of the arboretum newsletter, number 26. 15 Jan. 2012. <http://www.ncsu.edu/jcraulstonarboretum/publications/ newsletters/ncsu_arboretum_newsletters/News26_95-10.html >.

Rinehart, T.A., B.E. Scheffler, and S. Reed. 2006. Genetic diversity estimates for the genus Hydrangea and development of a molecular key based on SSR. J. Amer. Soc. Hort. Sci. 131:787-797.

Rinehart, T.A., R.N. Trigiano, P.A. Wadl, D. Hadziabdic, M.R. Pooler, and B.E. Scheffler. 2010. Characterization of eight microsatellite DNA markers for the native redbud tree (Cercis canadensis). Mol. Ecol. Resources 10:751-754.

Roethling, J.L. 2007. Eastern redbud plant named 'Hearts of Gold'. U.S. Plant Patent 17,740. U.S. Patent and Trademark Office, Washington, DC.

Rossetto, M. 2001. Sourcing of SSR markers from related plant species, p. 211-224. In: Henry, R.J. (ed.). Plant genotyping-The DNA fingerprinting of plants. CABI Publishing, New York, NY. 
Takezaki, N. and M. Nei. 1996. Genetic distances and reconstruction of phylogenetic trees from microsatellite DNA. Genetics 144:389-399.

Waldbieser, G.C., S.M. Quiniou, and A. Karsi. 2003. Rapid development of gene-tagged microsatellite markers from bacterial artificial chromosome clones using anchored TAA repeat primers. Biotechniques 35:976-979.

Weathington, M. 2009. The Cercis collection at the JC Raulston Arboretum. Public Gardens 24:38-39.

Werner, D.J. 2006. Breeding efforts in Cercis at North Carolina State University. Proc. Southern Nursery Assn. Res. Conf. 51:595-596.

Werner, D.J. and L.K. Snelling. 2010. 'Ruby Falls' and 'Merlot' redbuds. HortScience 45:146-147.
White, T.J., T. Bruns, S. Lee, and J. Taylor. 1990. Amplification and direct sequencing of fungal ribosomal RNA genes for phylogenetics, p. 315-322. In: Innis, M.A., D.H. Gelfand, J.J. Sninsky, and T.J. White (eds.). PCR protocols: A guide to methods and applications. Academic Press, San Diego, CA.

Zhang, D.X. 1994. Leaf venation of Cercideae (Leguminosae). J. Trop. Subtrop. Bot. 2:45-57.

Zhang, D.X. 1999. Scanning electron microscopic study of seed coat patterns in Cercis L. (Leguminosae). J. Trop. Subtrop. Bot. 7:34-36.

Zwickl, D.J. and D.M. Hillis. 2002. Increased taxon sampling greatly reduces phylogenetic error. Syst. Biol. 51:588-598. 